\title{
Liposome mitigation of curcumin inhibition of cardiac potassium delayed-rectifier current
}

\author{
Lawrence Helson' \\ George Shopp ${ }^{2}$ \\ Annie Bouchard ${ }^{3}$ \\ Muhammad Majeed ${ }^{4}$ \\ 'SignPath Pharma, Quakertown, PA, \\ USA; ${ }^{2}$ Shopp Nonclinical Consulting, \\ Boulder, CO, USA; ${ }^{3}$ IPS Therapeutique \\ Inc, Sherbrooke, Quebec, Canada; \\ ${ }^{4}$ Sabinsa Inc, Princeton, NJ, USA
}

This article was published in the following Dove Press journal:

Journal of Receptor, Ligand and Channel Research

15 November 2012

Number of times this article has been viewed

Background: The duration of the QT interval on the standard electrocardiogram (ECG) is measured from the beginning of the QRS complex (depolarization of the cardiac myocyte) to the end of the T-wave (completion of the repolarization phase of the cardiac myocyte). Repolarization is a result of currents generated by the outward flow of $\mathrm{K}^{+}$through the $\mathrm{K}^{+}$ channels. Obstruction of ion flow in the channel leads to delayed repolarization, evidenced by a prolonged QT interval. Clinically, this is known as the long QT syndrome (LQTS), which, when expressed, can lead to severe cardiac arrhythmias and sudden death. Obstruction of $\mathrm{K}^{+}$ion flow can result from gene mutations (eg, the human ether-a-go-go-related gene [hERG]) resulting in phenotypic abnormalities in $\mathrm{K}^{+}$channels and/or common structurally diverse drugs. These gene abnormalities or drug-induced changes result in decreased cardiac delayed-rectifier $\mathrm{K}^{+}$ current $\left(\mathrm{I}_{\mathrm{Kr}}\right.$, or KV11.1) in congenital or acquired LQTS, respectively. Increased risk of LQTS is a major drug development hurdle, and many drugs have been withdrawn during preclinical development, assigned black box warnings following approval, or withdrawn from the market. Autosomal recessive or dominant LQTS based upon 500 possible mutations in ten different genes coding for $\mathrm{K}^{+}$channels has an incidence of $1: 3000$ or about 100,000 persons in the USA. Prolonged QT intervals or risk of LQTS occurs in $2.5 \%$ of the asymptomatic US population. The probability of cardiac death in patients with asymptomatic congenital LQTS who are concomitantly medicated with LQTS-inducing drugs appears to have increased.

Methods: E-4031 (methanesulfanalide), terfenadine (Seldane ${ }^{\circledR}$ ), curcumin, liposomal curcumin, empty liposomes, empty liposomes vortexed with E-4031, or terfenadine and empty liposomes vortexed with curcumin were assayed for their effects on the $\mathrm{K}^{+}$-selective $\mathrm{I}_{\mathrm{Kr}}$ tail current inhibition using human embryonic kidney (HEK 293) cells stably transfected with the hERG gene via the whole-cell manual patch clamp technique.

Results: E-4031, terfenadine, and curcumin inhibit $\mathrm{I}_{\mathrm{Kr}}$ channel following nM-to- $\mu \mathrm{M}$ exposures. Empty liposomes had no effect on $\mathrm{I}_{\mathrm{Kr}}$. Both the liposomal curcumin formulation and vortexed mixtures of empty liposomes and curcumin prevented the $\mathrm{I}_{\mathrm{Kr}}$ inhibitory effect of curcumin in a dose-dependent manner. Empty liposomes vortexed with E-4031 prevented the effect of E-4031 to a lesser extent, while empty liposomes vortexed with terfenadine did not alter its $\mathrm{I}_{\mathrm{Kr}}$ inhibitory activity.

Conclusion: Curcumin causes an inhibition of the hERG tail current density. The liposomal curcumin formulation, as well as a mixture of empty liposomes with curcumin or with E-4031, blocked drug-induced $\mathrm{I}_{\mathrm{Kr}}$ inhibition. However, empty liposomes mixed with terfenadine did not alter terfenadine's $\mathrm{I}_{\mathrm{Kr}}$ inhibitory effects. The liposomes protected against the inhibitory effect of some compounds on the $\mathrm{K}^{+}$-selective $\mathrm{I}_{\mathrm{Kr}}$ current, independent of their potency.

Keywords: torsade de point, hERG, ion channel

Correspondence: Lawrence Helson SignPath Pharma, I 375 California Road, Quakertown, PA, I895I, USA

Tel + I 2155389996

Fax +I 2I5 538 I245

Email Ihelson@comcast.net
Journal of Receptor, Ligand and Channel Research 2012:5 I-8

(C) 2012 Helson et al, publisher and licensee Dove Medical Press Ltd. This is an Open Access article which permits unrestricted noncommercial use, provided the original work is properly cited.
Dovepress

http://dx.doi.org/10.2147/JRLCR.S37138 


\section{Introduction}

Ion channels are pore-forming integral membrane proteins that establish and control the electrochemical gradient across the plasma membrane. The channels are assembled as a circular arrangement of proteins packed around a water-filled pore. The ions pass through the channel, which may be open or closed by chemicals, electrical signals, temperature, or mechanical force. Ion channel dysfunction is associated with gene mutation, or with drugs interfering with ion flow, or both.

Dysfunctions in cardiac electrolyte $\mathrm{K}^{+}$, calcium, and sodium channels in the cardiac myocyte membrane induce defects in the electrical currents and normal action potentials necessary for coordinated myocyte contraction and ultimately for maintenance of normal blood circulation.

The central roles of the 40 members and 12 subfamilies of voltage-gated $\mathrm{K}^{+}$channels (Kvs) are to repolarize cell membranes following action potentials. Levels of depolarization and repolarization are modulated by the flux of $\mathrm{K}^{+}$ions in the cardiac myocyte $\mathrm{K}^{+}$channels. Congenital and/or drug-induced channel defects may be associated with a risk of morbidity and mortality in otherwise asymptomatic individuals. The channel proper, coded by the gene $\mathrm{KCNH} 2$ or human ether-a-go-go-related gene ( $\mathrm{hERG}$ ), contains proteins designated Kv11.1 and the Lv11.1 $\alpha$-subunit of the rapidly activating rectifier $\mathrm{K}^{+}$current, or cardiac delayed-rectifier $\mathrm{K}^{+}$current $\left(\mathrm{I}_{\mathrm{Kr}}\right.$, or KV11.1). This cell membrane-located channel mediates the "rapid" delayed-rectifier current $\mathrm{I}_{\mathrm{Kr}}$ by conducting $\mathrm{K}^{+}$ions out of the cardiac myocytes, allowing repolarization, which is a critical mechanism to allow the cardiac potential to return to the resting state.

Even though the three-dimensional structure of the hERG channel is unknown, insight into its structure has been gained from site-directed mutagenesis data. ${ }^{1}$ Within the hERG channel pore cavity, ion flux and currents can be modified, depending upon the open or closed states, and by drug interactions at key high-affinity drug-binding sites. These sites are the aromatic amino-acid residues (Y652 and F656) on the inner helices of the pore. The most important currents mediated by drugs, the sensitive delayed $\mathrm{I}_{\mathrm{Kr}}$ (rapid) current which repolarizes the myocardial cells and the $\mathrm{I}_{\mathrm{Ks}}$ (slow) rectifier currents, are exhibited on the standard ECG as the QT interval which, when corrected for heart rate, is conventionally defined as QTc.

Congenital defects in ion channels, first described in 1957, alter the balance of currents determining repolarization of the action potential and predispose the patient to long QT syndrome (LQTS) arrhythmias and sudden cardiac death. ${ }^{2}$ Mutations have been identified that give rise to subtypes of congenital LQTS, familial arrhythmogenic syndromes characterized by abnormal ion channel function, delayed repolarization, a prolonged QT interval on the electrocardiogram, and a life-threatening polymorphic ventricular tachycardia known as torsade de pointes (TdP). Different mutations in the hERG gene and its coded proteins translate to defects in channel function and a number of clinical syndromes. Type 2 congenital long-QT syndrome (LQT2) results from A614V missense mutations in the $\mathrm{KCNH}$ gene. It is characterized by four classes of loss of Kv11.1 protein and consequent channel dysfunction. These abnormal Kv11.1 channels include: (Class 1) a dominant-intracellular trafficking-deficient ion-channel protein, usually due to missense mutations; (Class 2) a correctible phenotype when cells are incubated for 24 hours at $27^{\circ} \mathrm{C}$ temperature, or with exposure to the drug E-4031; (Class 3) channel gating; and (Class 4 ) permeation. ${ }^{4}$ Blockade by any of these, and particularly the "rapid" current, prolongs the action potential and manifests on the ECG as a prolonged QT interval and the emergence of other T- or U-wave abnormalities. Under such circumstances, activation of an inward depolarization current induces increased dispersion of repolarization. The latter results in a heterogeneous recovery of excitability and induction of TdP, an early premature ventricular contraction. This is where ventricular depolarization, ie, the R-wave, occurs simultaneously with the relative refractory period at the end of repolarization (the latter half of the T-wave) and initiates pathologic T-U waves and Tdp. Sustained TdP leads to a zone of functional refractoriness in the myocardium and to cardiac arrhythmias. The ECG reading in TdP exhibits a rapid polymorphic ventricular tachycardia with a characteristic twist of the QRS complex around the isoelectric baseline. This is characterized by a rotation of the heart's electrical axis by as much as $180^{\circ}$ and long and short RR intervals, and clinically leads to a fall in arterial blood pressure, syncope, and degeneration into ventricular fibrillation and sudden death.

On the ECG, retardation of the $\mathrm{I}_{\mathrm{Kr}}$ current interval is synonymous with QT prolongation when greater than $440 \mathrm{~ms}$ in men and $460 \mathrm{~ms}$ in women. Pharmacological inhibition of hERG $\mathrm{K}^{+}$channels by structurally and therapeutically diverse drugs translates to the clinical acquired form of the LQTS. While QT-prolonging drugs represent two to three percent of the total prescriptions in the developed world, the reported incidence of QT prolongation and dosage varies significantly within different drug classes. The latter include Class 1A and Class III antiarrhythmics, antihistamines, antimicrobials, antipsychotics, tricyclic antidepressants, 
prokinetics, and antianginals. Recently, curcuminoids were reported to block human cardiac $\mathrm{K}^{+}$channels. ${ }^{5}$

Increased incidence of QT prolongation may also occur in the presence of hypomagnesemia, hypokalemia, hypocalcemia, hypoxia, acidosis, heart failure, left ventricular hypertrophy, slow heart rate, female gender, hypothermia, and subarachnoid hemorrhage. The severity of arrhythmia at a given QT interval, and development of TdP, varies from drug to drug and patient to patient, and may not be linearly related to the dose or plasma concentration of a specific drug. However, antiarrhythmic cardiac drugs affecting the $\mathrm{K}^{+}$(Class III) and non-cardiac drugs that significantly alter repolarization, as measured by prolongation of the QT interval, predispose the patient to TdP. Additional factors associated with an increased tendency toward TdP include familial LQTS. The most common causes of familial LQTS are mutations in genes.

$K C N Q 1$ codes for KvLTQ1, the alpha subunit of the slow delayed $\mathrm{K}^{+}$rectifier channel that is highly expressed in the heart. The current through the heteromeric channel when interacting with the minK beta subunit is known as $\mathrm{I}_{\mathrm{Ks}}$. When missense-mutated, it reduces the amount of repolarizing current needed to terminate the action potential. These LTQ1 mutations represent $35 \%$ of all cases, and are the least severe, usually causing syncope.

$K C N H 2$, or the hERG gene, when mutated represents an additional $30 \%$ of all genetic cases, and is the subunit of the rapid delayed rectifier $\mathrm{K}^{+}$channel hERG + MiRP1. Current through this channel, known as the $\mathrm{I}_{\mathrm{Kr}}$ channel, is responsible for the termination of the action potential and the length of the QT interval. When reduced, it leads to LQT2. The rapid current is not only the most drug sensitive, but is also associated with the pro-arrhythmic effect in HisPurkinje cells and M cells in the midventricular myocardium. Drug-induced LQTS occurs with antiarrhythmic drugs, antihistamines, antipsychotics, and other compounds. The combination of genetic LQTS and LQTS-inducing drugs increases susceptibility to lethal side effects. Most drugs causing LQTS block the $\mathrm{I}_{\mathrm{Kr}}$ current via the hERG gene. This channel exhibits unintended drug binding at tyrosine 652 and phenylalanine 656 which, when bound, block ion-induced current conduction.

Uncommon but lethal mutations in gene $S C N 5 A$ slow inactivation of the alpha subunit of the sodium channel, prolonging $\mathrm{Na}^{+}$influx and the current $\mathrm{I}_{\mathrm{Na}}$ during depolarization. Continued depolarizing current through the channel late in the action potential induces a late bursting current.
L-type calcium channels reopen during the plateau phase of the action potential following LQTS as "early after depolarizations." Their activity is sensitive to adrenergic stimulation and increases the risk of sudden death during adrenergic states in the presence of impaired repolarization.

In these subjects, $\mathrm{TdP}$ can be precipitated subsequent to exercise or by emotional surprise unrelated to drugs. There are additional uncommon and rare mutations designated LQT4-13.

Apart from heart rate, the QT duration varies with recording and measurement techniques, sympatho-vagal activity, drugs, electrolyte disorders, cardiac or metabolic diseases, diurnal variation, and genetic LQT2 mutations. These parameters cause the reported incidence of drug-induced $\mathrm{TdP}$ to be loosely associated with clinical studies during drug development, post-marketing surveillance, epidemiologic studies, and anecdotal case reports. Detection of QT prolongation during preclinical drug development can lead to abandonment, and precludes any all-inclusive accounting of the actual incidence of drug-related QT prolongation. ${ }^{6}$ A number of QT-prolonging drugs have been withdrawn either during development or after being on the market. These include terfenadine, astemizole, gripafloxacin, terodilene, droperidole, lidoflazine, levomethadyl, sertindoyle, and cisapride $\left(\right.$ Prepulsid $\left.{ }^{\circledR}\right)$. Genetic and age-related susceptibility includes patients with structural heart disease, those taking hepatic C450 inhibitors, and those with a genetic predisposition or DNA polymorphisms. Older females are generally more susceptible than young females, while young males have increased susceptibility compared to elderly males.

Based on a drug-development requirement and on the previous publication of a curcuminoid mixture inhibiting $\mathrm{I}_{\mathrm{Kr}}{ }^{5}$ this study was initially undertaken to test synthesized curcumin (diferuloylmethane), the curcuminoid molecule responsible for $\mathrm{I}_{\mathrm{Kr}}$ inhibitory effects. The second objective was to determine whether the liposomal curcumin formulation would mitigate curcumin-induced $\mathrm{I}_{\mathrm{Kr}}$ inhibitory effects. The third objective was to determine if empty liposomes briefly (10 minutes) vortexed with curcumin or known specific $\mathrm{I}_{\mathrm{Kr}}$ inhibitors (E-4031 and terfenadine) could interfere with their $\mathrm{I}_{\mathrm{Kr}}$-blocking activity.

\section{Methods}

\section{Manual patch clamp assay}

Whole cell patch-clamp recordings were made at $35^{\circ} \mathrm{C} \pm 2^{\circ} \mathrm{C}$ from human embryonic kidney (HEK 293) cells stably transfected with hERG gene, maintained in culture with selective agent (G148; Sigma-Aldrich, St Louis, MO). Cells, 
plated onto $35 \mathrm{~mm}$ petri dishes, were washed twice with $1 \mathrm{~mL}$ of hERG external solution containing $140 \mathrm{mM} \mathrm{NaCl}$, $5 \mathrm{mM} \mathrm{KCl}, 1.8 \mathrm{mM} \mathrm{CaCl}_{2}, 1 \mathrm{mM} \mathrm{MgCl}, 10 \mathrm{mM}$ glucose, and $10 \mathrm{mM}$ HEPES ( $\mathrm{pH} 7.4$ ), followed by the addition of $2 \mathrm{~mL}$ of hERG external solution. The petri dish (experimental chamber) was mounted on the stage of an inverted phase contrast microscope. A borosilicate glass micropipette (open resistance of 5-15 M $\Omega$ ) pulled with a PMP-102 Programmable Micropipette Puller and filled with internal pipette solution containing $140 \mathrm{mM} \mathrm{KCl,} 1 \mathrm{mM} \mathrm{MgCl}$, 5 mM EGTA, $10 \mathrm{mM}$ HEPES, $4 \mathrm{mM} \mathrm{Mg-ATP,} \mathrm{and} 10 \mathrm{mM}$ sucrose was positioned above a single cell using an Eppendorf PatchMan micromanipulator. The micropipette was lowered to the cell until a close contact was achieved. The wholecell configuration was then obtained by applying a slight negative pressure (resistances were measured by a $5-\mathrm{mV}$ square pulse, to be in the G $\Omega$ range). Cell capacitance was immediately measured in order to evaluate cell surface area, using a conversion factor of $1 \mathrm{pF} / \mathrm{m}^{2}$. This cell surface area was later used to calculate net current density (as opposed to current amplitude).

Currents were acquired at a rate of $1 \mathrm{kHz}$ and were filtered using a low-pass 4-pole Bessel filter with the cutoff rate set at $500 \mathrm{~Hz}$. Baseline condition currents were recorded using the PClamp 10 acquisition suite (Molecular Devices, Sunnyvale, CA) following a 2-minute equilibration period. Currents were then recorded after 5 minutes of exposure to each concentration of test articles or positive controls (curcumin, liposomes, liposomal curcumin, curcumin + liposomes, terfenadine, terfenadine + liposomes, E-4031, E-4031 + liposomes). The cells were stimulated continuously (Figure 1).

Currents were analyzed using the Clampfit 10.2.0.14 module of the PClamp 10.2.0.14 software (Axon Instrument Inc [Molecular Devices], Foster City, CA).

For current rundown and solvent effect correction, all data points were corrected for solvent effect and time-dependent current rundown. Current rundown and solvent effects were measured simultaneously by applying the experimental design in test-article-free conditions (dimethyl sulfoxide

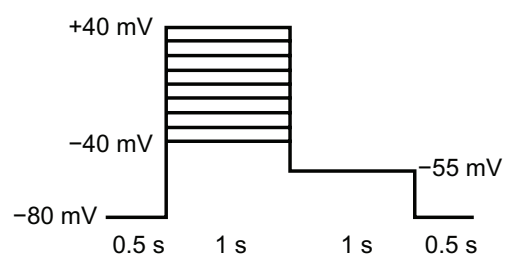

Figure I The cells were stimulated continuously. vehicle) over the same time frame, as was done with the test articles. The loss in current amplitude measured during these so-called vehicle experiments (representing both solvent effects and time-dependent rundown) was subtracted from the loss of amplitude measured in the presence of the test article, to isolate the effect of the test article apart from the effect of the solvent and the inevitable rundown in current amplitude over time.

\section{Statistical analysis}

Repeat Student's $t$-tests were used to assess statistical significance. For the test articles and positive controls, the currents recorded after exposure to the different concentrations were statistically compared to the currents recorded under baseline conditions. Currents recorded after the washout were statistically compared to the currents measured after the highest concentration of test articles. Statistical significance was considered to be $P \leq 0.05$.

\section{Chemicals}

Terfenadine $\left(\right.$ Seldane $^{\circledR}$ ), an antihistamine prodrug for the active form of fexofenadine, and E-4031 were selected as positive control compounds for this study. Terfenadine has reported ventricular arrhythmia cardiotoxic effects, particularly if taken in combination with macrolide antibiotics or ketoconazole. E-4031, a class III antiarrhythmic drug, is a synthetic toxin used solely for research purposes with one clinical exception. ${ }^{7}$ Its mechanism of action is to block the hERG voltage-gated $\mathrm{K}^{+}$channels. Terfenadine and E-4031 were both purchased from Sigma-Aldrich. Curcumin, 99.2\% pure, was synthesized under good manufacturing practice (GMP) conditions in December 2010 by Sami Labs (Bangalore, India). The concentrations of curcumin (0.014, $0.2,3.4$, and $11.4 \mu \mathrm{M}$ ) were based on information available at the time of the design of this study. The concentrations were selected based on: (1) the predicted human plasma levels at the planned lowest Phase 1 dose level, (2) the predicted human plasma concentrations at the planned highest Phase 1 dose level, (3) a concentration being 30-fold over the predicted human therapeutic plasma levels, and (4) a concentration being 100-fold over the predicted human therapeutic plasma levels. These selected concentrations are considered to provide valuable predictions of the effect of curcumin on human cardiac electrophysiology.

The GMP-grade liposomal curcumin was produced on March 31, 2011 at Polymun GmbH (Vienna, Austria). The empty liposomes were also obtained from Polymun $\mathrm{GmbH}$. The liposomes were made up of a 9:1 ratio of DMPC 
(1,2-dimyristoyl-sn-glycero-3-phosphocholine) and DMPG (1,2-dimyristoyl-sn-glycero-3-phospho-rac-[1-glycerol]).

\section{Results}

Curcumin, at a concentration of $11.4 \mu \mathrm{M}$, caused $80 \%$ of inhibition of the hERG tail current density at $\mathrm{I}_{+20}(\mathrm{n}=7)$ (Figure 2). Paired Student's $t$-tests confirmed that the difference in normalized current density measured at baseline and in the presence of $0.2-11.4 \mu \mathrm{M}$ of curcumin reached the selected threshold for statistical significance $(P \leq 0.05)$. The liposomal curcumin formulation, at the same curcumin concentration of $11.4 \mu \mathrm{M}$, induced an inhibition of the hERG tail current at $I_{+20}(n=7)$ of $21 \%$, which was statistically less than the inhibition induced by the curcumin alone. When liposomes were vortexed for 10 minutes at room temperature with curcumin at a ratio of $9: 1$, the same curcumin concentration $(11.4 \mu \mathrm{M})$ caused an inhibition of the hERG current density of $45 \%(n=3)$. The liposomes alone did not cause any inhibition of the hERG tail current density for $\mathrm{I}_{+20}(\mathrm{n}=3)$.

E-4031, at concentrations of $30 \mathrm{nM}, 100 \mathrm{nM}$, and $300 \mathrm{nM}$, caused an inhibition of the hERG tail current density for $I_{+20}(n=3)$ of $32 \%, 66 \%$, and $82 \%$, respectively (Figure 3 ). Paired Student's $t$-tests confirmed that the difference in normalized current density measured at baseline and in the presence of 30-300 nM of E-4031 reached the selected threshold for statistical significance $(P \leq 0.05)$. When E-4031 was vortexed with empty liposomes for 10 minutes at room temperature at a ratio of 9:1, the same E-4031 concentrations (30 nM, $100 \mathrm{nM}$, and $300 \mathrm{nM}$ ) caused an inhibition of the hERG current density, but to a lesser extent, of $22 \%, 31 \%$, and $60 \%$, respectively $(n=3)$. Non-paired Student's $t$-tests confirmed that the difference between the effect of E-4031 alone at $100 \mathrm{nM}$ and of E-4031 at the same concentration vortexed with empty liposomes was statistically significant. The difference between the effect of E-4031 alone and of E-4031 vortexed with empty liposomes was not statistically significant at $300 \mathrm{nM}$. The lack of statistical significance at $300 \mathrm{nM}$ was likely due to the higher variability of the E-4031 vortexed with empty liposomes at this concentration.

Terfenadine, at concentrations ranging from $30 \mathrm{nM}$ to $300 \mathrm{nM}$, caused an inhibition of the hERG tail current density for $\mathrm{I}_{+20}(\mathrm{n}=3)$ of $45 \%-88 \%$ (Figure 4). Paired Student's $t$-tests confirmed that the difference in normalized current density measured at baseline and in the presence of 100-300 nM of terfenadine reached the selected threshold for statistical significance $(P \leq 0.05)$. When terfenadine was vortexed with empty liposomes for 10 minutes at room temperature at a ratio of 9:1, the same terfenadine concentrations (30-300 nM) caused corresponding inhibition of hERG current density $(61 \%-79 \%)(n=3)$. Non-paired Student's $t$-tests confirmed that the difference between the effect of terfenadine alone and terfenadine at the same concentrations vortexed with empty liposomes was not statistically significant. Table 1 shows the $\mathrm{IC}_{50}$ for each of the treatments.

\section{Discussion}

Curcumin, within the range of concentrations tested $(0.2-11.4 \mu \mathrm{M})$ and in the specific context of this study, induced an inhibition of the $\mathrm{I}_{\mathrm{Kr}}$ current; ie, it interacts with the proteins encoded by the $\mathrm{hERG}$ gene (the pore-forming alpha subunit of the $\mathrm{K}^{+}$channel). The more rapid inactivation of the channel translated into a shorter open time for the channel,
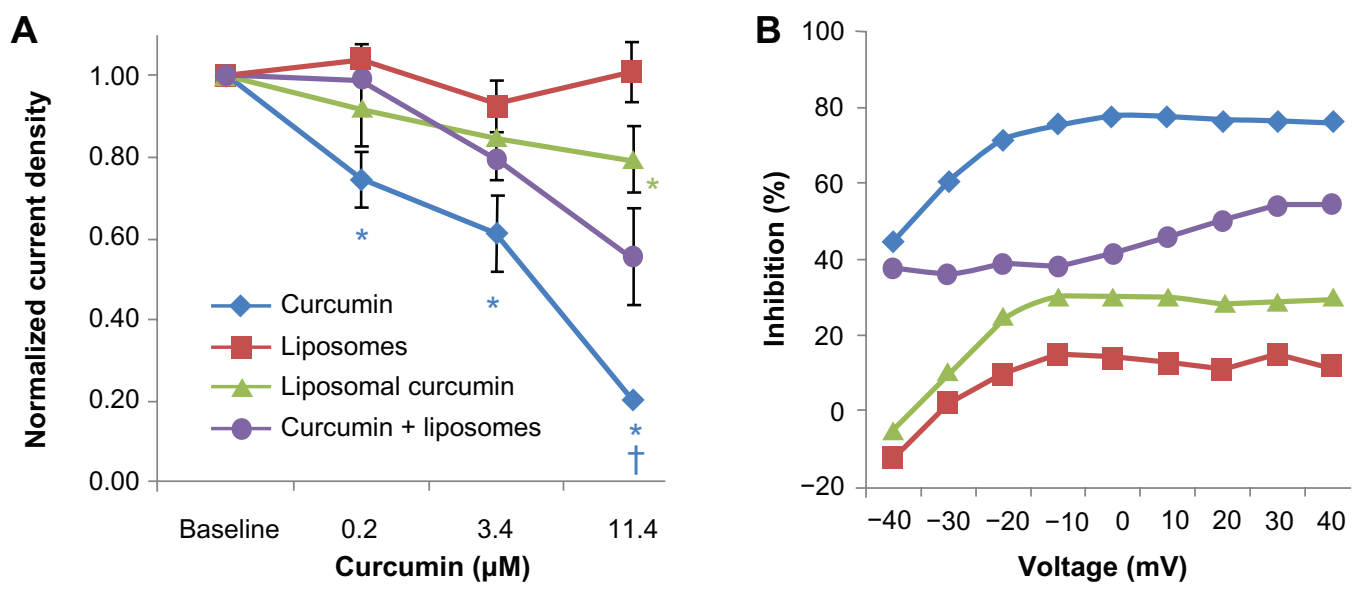

Figure 2 hERG tail current density averages obtained by measuring the hERG tail peak amplitude at $20 \mathrm{mV}$ in baseline conditions and in the presence of curcumin, liposomal curcumin, liposomes alone, or liposomes vortexed with curcumin (9:I ratio). The curcumin concentrations were always 0.2 , 3.4, and II.4 $\mu$ M. (A) Current density was measured from seven cells, averaged, normalized against baseline current density, and corrected for time and solvent effects. Statistical comparisons between postdrug exposure and baseline current density levels were made using repeat paired Student's $t$-tests $(*)$. Differences were considered significant when $P \leq 0.05$. (B) Voltage dependency of the hERG tail current inhibition at the higher concentration of curcumin tested (II.4 $\mu \mathrm{M})$. 

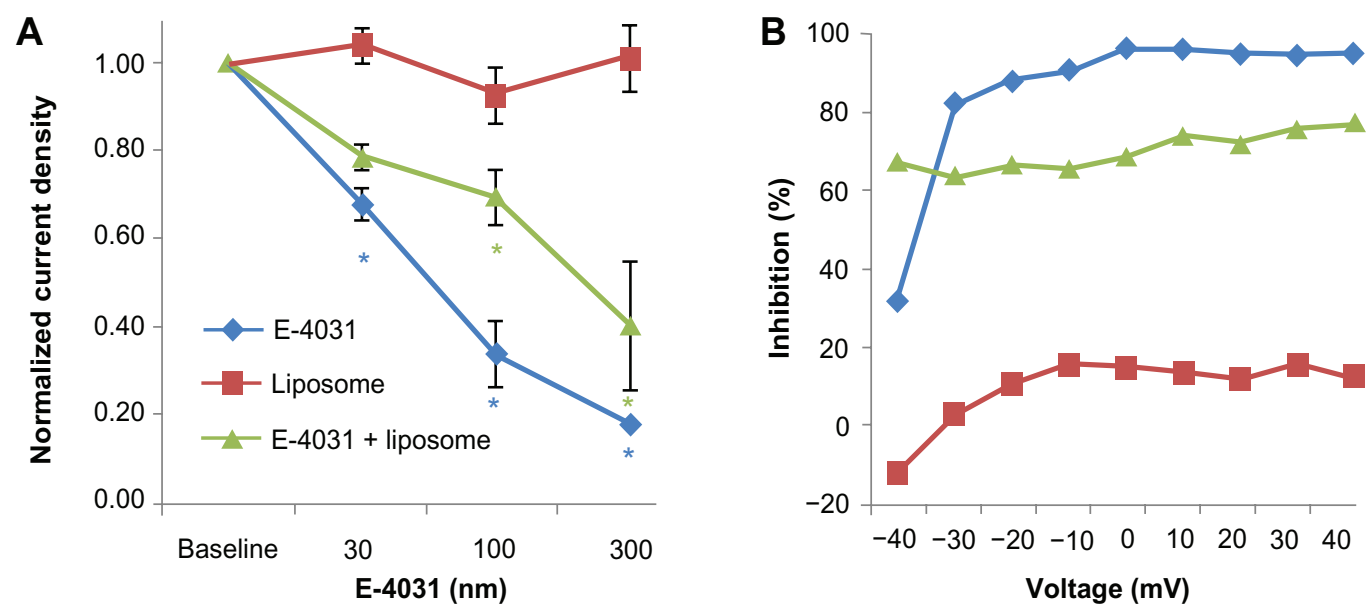

Figure 3 hERG tail current density averages obtained by measuring the hERG tail peak amplitude at $20 \mathrm{mV}$ in baseline conditions and in the presence of E-403I, liposomes alone, or liposomes vortexed with E-403I (9:I ratio). The E-403I concentrations were always $30 \mathrm{nM}, 100 \mathrm{nM}$, and $300 \mathrm{nM}$. (A) Current density was measured from three cells, averaged, normalized against baseline current density, and corrected for time and solvent effects. Statistical comparisons between post-drug exposure and baseline current density levels were made using repeat paired Student's $t$-tests $(*)$. Differences were considered significant when $P \leq 0.05$. (B) Voltage dependency of the hERG tail current inhibition at the higher concentration of E-403I tested (300 nM).

which led to decreased ion flow across the membrane. It was not possible, based on the experimental dataset, to confirm whether the changes in gating kinetics were due to a direct interaction of curcumin with the pore-forming peptides, or whether a change in lipid packing in proximity to the hERG channel was the cause of the more rapid inactivation kinetics, as exemplified by the terfenadine sensitization.

Dimethyl sulfoxide, for example, changes the fluidity of the lipid membrane into which the channel is embedded, leading to faster activation and inactivation kinetics of the channels. On the other hand, there are numerous examples of molecules that alter the inactivation gates of the hERG channel via direct interaction with the peptide, including oxygen peroxide and tetraethylammonium.

A

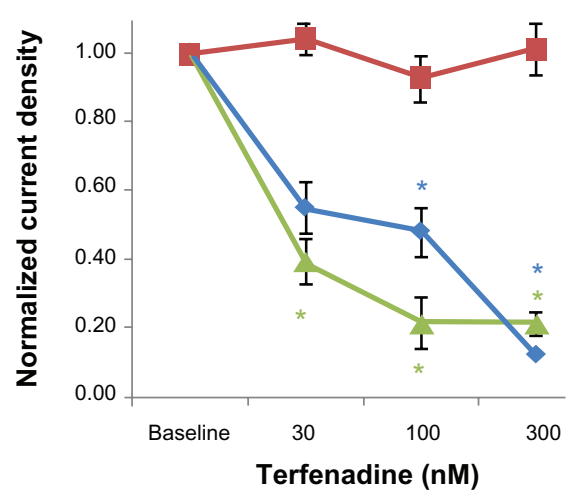

Moha ou Maati et al reported similar inhibition of the hERG current with a curcuminoid mixture ( $78 \%$ curcumin $){ }^{5}$ The results of the present study support their initial observation and emphasize that the curcumin (diferuloylmethane) molecule exhibits the main portion, if not the entirety, of the $\mathrm{I}_{\mathrm{Kr}}$ inhibition.

The present study demonstrated that liposomes may protect against the inhibitory effect of compounds on the $\mathrm{K}^{+}$-selective $\mathrm{I}_{\mathrm{Kr}}$ current generated under normoxic conditions using stably transfected human embryonic kidney (HEK 293) cells. Compared to initial observations with curcumin, it was demonstrated that liposomal curcumin or vortexed mixtures of empty liposomes with curcumin ameliorated $\mathrm{I}_{\mathrm{Kr}}$ inhibition by curcumin. The same experiment was repeated

B

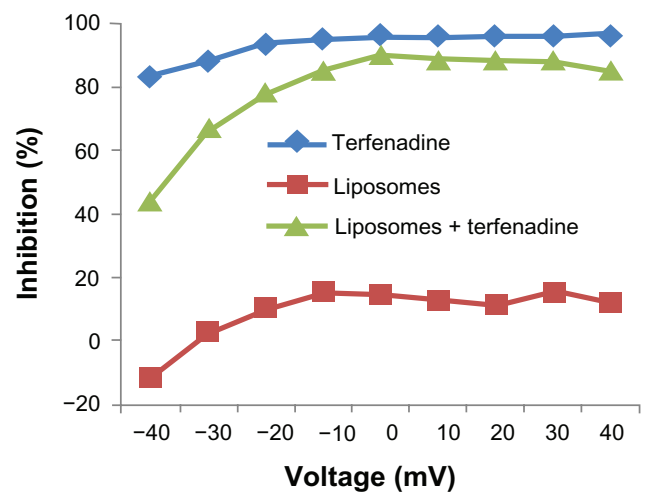

Figure 4 hERG tail current density averages obtained by measuring the hERG tail peak amplitude at $20 \mathrm{mV}$ in baseline conditions and in the presence of terfenadine, liposomes alone, or liposomes vortexed with terfenadine (9:I ratio). The terfenadine concentrations were always $30 \mathrm{nM}, 100 \mathrm{nM}$, and $300 \mathrm{nM}$. (A) Current density was measured from three cells, averaged, normalized against baseline current density, and corrected for time and solvent effects. Statistical comparisons between post-drug exposure and baseline current density levels were made using repeat paired Student's $t$-tests $(*)$. Differences were considered significant when $P \leq 0.05$. (B) Voltage dependency of the hERG tail current inhibition at the higher concentration of terfenadine tested $(300 \mathrm{nM})$. 
Table I Concentration that causes $50 \%$ inhibition $\left(\mathrm{IC}_{50}\right)$ of the hERG current density

\begin{tabular}{ll}
\hline Compounds & IC $_{50}$ \\
\hline Curcumin & $4.9 \mu \mathrm{M}$ \\
Liposomal curcumin & $\mathrm{n} / \mathrm{a}^{\mathrm{a}}$ \\
Curcumin (liposomes + curcumin) & $\mathrm{n} / \mathrm{a}^{\mathrm{a}}$ \\
Liposomes & $\mathrm{n} / \mathrm{a}^{\mathrm{a}}$ \\
E-403I & $56 \mathrm{nM}$ \\
E-403I (liposomes + E-403I) & $210 \mathrm{nM}$ \\
Terfenadine & $61 \mathrm{nM}$ \\
Terfenadine (liposomes + terfenadine) & $20 \mathrm{nM}$ \\
\hline
\end{tabular}

Note: a ${ }^{a} C_{50}$ was not reached within the range of concentrations tested $(0.2-1 \mathrm{I} .4 \mu \mathrm{M})$.

with E-4031, a well-known $\mathrm{I}_{\mathrm{Kr}}$ inhibitor. Similar results were obtained when liposomes were vortexed with E-4031. This suggests that liposome encapsulation of compounds, or just in the presence of liposomes, prevents interactions of active compounds with channel drug-receptor sites, allowing more normal gating kinetics to occur. The mechanism of action of liposomes appears to be compound-specific but is independent of potency, since there was no protective effect of liposomes on terfenadine inhibition of $\mathrm{I}_{\mathrm{Kr}}$ in this in vitro model system.

In the absence of broader studies, this one does not preclude the possibility that liposomes interfere with other curcuminoids, similarly structured molecules or classes of hERG active agents other than E-4031. In support of this, other liposomal products have been shown to have a protective function against agents inducing QT prolongation. ${ }^{8}$ That this particular empty liposome did not appear to interact with the protein encoded by the hERG gene in the absence of curcumin, or in the presence of E-4031 and terfenadine, raises issues about the specificity and degree of affinities or preferential interactions for the receptors in the $\mathrm{K}^{+}$channel. ${ }^{9}$ There are previous reports of similar empty liposomes composed of DMPC and DMPG (7:3) that lacked general toxicity and decreased nystatin toxicity. ${ }^{10}$ Based on these data, it does not appear necessary to synthesize a formulated liposomal compound to mitigate or abrogate $\mathrm{I}_{\mathrm{Kr}}$ suppression by curcumin or other QT-prolonging agents. A simple mixing of the compound with the liposomes may be sufficient.

\section{Conclusion}

The inhibition of the $\mathrm{I}_{\mathrm{Kr}}$ current induced by curcumin and E-4031 is mitigated when the curcumin is incorporated within a liposome or simply vortexed with liposomes prior to exposure. The E-4031 effects are mitigated to a lesser extent, compared to curcumin, by vortexing with empty liposomes.
In contrast, terfenadine-induced $\mathrm{I}_{\mathrm{Kr}}$ suppression is not inhibited when vortexed with empty liposomes. Combined intravenous administration of these liposomes with QT-prolonging drugs may mitigate delayed QT in vivo. For orally administered PQTS drugs, a concurrent subcutaneous administration of an extended-release formulation of liposomes may suffice. This will need to be tested with QT-prolonging drugs in in vivo animal models of QT-prolongation. Where simple mixing is not effective, liposomal formulations of QT-prolonging drugs may be warranted.

\section{Disclosure}

Lawrence Helson is the CEO and president of SignPath Pharma Inc, which is developing liposomal curcumin. This paper is the result of an FDA requirement that during commercial preclinical drug development, all drugs be tested for potential cardiac toxicity. The study is derived from an FDA requirement for an investigational drug application, but, in our view, it has academic implications that should be in the public domain. Were it not for the authors' commercial connections and direct or indirect conflicts of interest, these data would not exist. Dr Majeed, CEO of Sabinsa Inc, freely supplied the synthesized drug. Ms Bouchard ran the assays for IPS therapeutics Inc. Dr G Shopp of Shopp Consulting helped design the study and contributed to the writing. L Helson MD, CEO of SignPath Pharma, was responsible for the overall drug development. Based upon the unanticipated observation of hERG protection by the liposome formulation and empty liposomes, it is our opinion that these data should be reported in the literature, with the admission that there do exist conflicts of interest on the part of the authors.

\section{References}

1. Stansfeld PJ, Gedeck P, Gosling M, Cox B, Mitcheson JS, Sutclif MJ Drug block of the hERG potassium channel: insight from modeling. Proteins. 2007;68(2):568-580.

2. Jervell A, Lang-Nielson F. Congenital deaf-mutism, functional heart disease with prolongation of the QT interval and sudden death. Am Heart J. 1957;54:59-68.

3. Zhou Z, Gong Q, January CT. Correction of defective protein trafficking of a mutant HERG potassium channel in human long QT syndrome: pharmacological and temperature effects. J Biol Chem. 1999;274: 31123-31126.

4. Anderson CL, Delisle BP, Anson BD, et al. Most LQT2 mutations reduce Kv11.1 (hERG) current by a class 2 (trafficking deficient) mechanism. Circulation. 2006;113:365-373.

5. Moha ou Maati H, Ducroq J, Rivet J, Faivre JF, Le Grande M, Bois P. Curcumin blocks the recombinant human cardiac KCNQ1/KCNE1 channels (IKs) stably expressed in HEK 293 cells. Congress de Physiologie de Pharmacologie et de Therapeutique; April 9-11, 2008; ClermontFerrand, France. Fund Clin Pharmacol. 2008;22(Suppl 1):1-102.

6. Yap YG, Camm AJ. Drug induced QT prolongation and torsades de pointes. Heart. 2003;89:1363-1372. 
7. Okada T, Ogawa S, Sadanaga T, Mitamura H. Assessment of reverse usedependent blocking actions of class III antiarrhythmic drugs by 24-hour Holter electrocardiography. J Am Coll Cardiol. 1996;27:84-89.

8. Maciel NR, Reis PG, Kato KC, et al. Reduced cardio-vascular alterations of tarter emetic administered in long-circulating liposomes in rats. Toxicol Lett. 2010;199(3):234-238.

9. Zachariae U, Giordanetto F, Leach AG. Side chain flexibilities in the human ether-a-go-go related potassium channel (hERG) together with matched-pair binding studies suggest a new binding mode for channel blockers. J Med Chem. 2009;52(14):4266-4276.
10. Mehta RT, Hopfer RL, Gunner LA, Juliano RL, Lopez-Berestein G. Formulation, toxicity, and antifungal activity in vitro of liposomalencapsulated nystatin as therapeutic agent for systemic candidiasis. Antimicrob Agents Chemother. 1987;31(12):1897-1900.

\section{Publish your work in this journal}

The Journal of Receptor, Ligand and Channel Research is an international, peer-reviewed, open access, online journal. The journal welcomes laboratory and clinical findings in the fields of biological receptors, ligands, channel and signal transduction research including: receptors and signalling; ligands; transporters, pores and channels; binding and activation; receptor regulation; role of receptors in diseases and their treatment; molecular basis of membrane structure and functions; molecular models of membranes. The manuscript management system is completely online and includes a very quick and fair peer-review system. Visit http://www.dovepress.com/ testimonials.php to read real quotes from published authors.

Submit your manuscript here: http://www.dovepress.com/journal-of-receptor-ligand-and-channel-research-journal 